\title{
L'antropologia duale come imago Dei
}

\author{
A antropologia dual como imago Dei
}

\author{
Dual anthropology as imago Dei
}

\author{
Angela Ales Bello \\ Pontificia Università Lateranense \\ Itália
}

\begin{abstract}
Due sono i contributi importanti di Edith Stein rispetto al tema che s'intende trattare: 1) la sua proposta antropologica che sintetizza, in modo geniale, l'analisi fenomenologia dell'umano inaugurata da Edmund Husserl e il contributo della filosofia medievale attraverso la lettura, in particolare, dei testi di Agostino e Tommaso d'Aquino; 2) la sua riflessione personale sulla questione del femminismo, che si andava diffondendo al suo tempo in Germania, riflessione che sfocia nella sua teorizzazione di quella che definisco $l^{\prime \prime \prime}$ antropologia duale". Nell'indagare l'essere umano maschile e femminile la Stein si pone da diversi punti di vista: psicologico, sociologico, storico, filosofico e anche teologico, cosa che la consente di esaminare in modo completo un fenomeno complesso che investe i costumi umani, che ha inciso per millenni nella storia dell'umanità e che continua a essere presente, cioè la sottomissione del femminile. Ella propone, al contrario, l'uguaglianza fra i due pur nella differenza. Ricercando le origini di tale fenomeno dal punto di vista teologico Edith Stein si rifà alle Sacre Scritture della tradizione ebraico cristiana e rilegge il contributo del Genesi e l'azione di Redenzione di Gesù Cristo. La sua è una proposta che deve essere ritenuta veramente costruttiva e, a mio avviso, una de le più importanti nell'ambito degli studi di genere; in essa si può trovare una critica convincente alle teorie deboliste della cultura contemporanea.
\end{abstract}

Parole chiave: antropologia duale; femminismo; fenomenologia; Edith Stein

\section{Resumo}

Há duas importantes contribuições de Edith Stein para o tema que se pretende tratar: 1) a sua proposta antropológica que sintetiza, de forma brilhante, a análise fenomenológica do ser humano inaugurada por Edmund Husserl e a contribuição da filosofia medieval através da leitura, em particular, dos textos de Agostinho e Tomas de Aquino; 2) sua reflexão pessoal sobre a questão do feminismo, que se difundia em sua época na Alemanha, uma reflexão que leva à sua teorização do que defino como "antropologia dual". Ao investigar o ser humano masulino e feminino, Stein se coloca em diferentes pontos de vista: psicológico, sociológico, histórico, filosófico e até teológico, o que lhe permite analisar de forma abrangente um fenômeno complexo que envolve os costumes humanos, que incide há milênios na história da humanidade e que continua presente: a submissão do feminino. Ela propõe, ao contrário, a igualdade entre os dois, mesmo na diferença. Pesquisando as origens deste fenômeno do ponto de vista teológico, Edith Stein refere-se às Escrituras da tradição judaico-cristã e relê a contribuição do Gênesis e a ação da Redenção de Jesus Cristo. É uma proposta que deve ser considerada realmente construtiva e, em nosso entendimento, uma das mais importantes no campo dos estudos de gênero; nela se pode encontrar uma crítica convincente a teorias inconsistentes da cultura contemporânea.

Palavras-chave: antropologia dual; feminismo; fenomenologia; Edith Stein 


\begin{abstract}
There are two important contributions of Edith Stein to the subject of this article: 1) her anthropological proposal which brilliantly synthesizes the phenomenological analysis of the human being inaugurated by Edmund Husserl and the contribution of medieval philosophy through the reading, in particular, of the texts of Augustine and Thomas Aquinas; 2) her personal reflection on the question of feminism, which was spreading in her time in Germany, a reflection that leads to her theory of which I define as a "dual anthropology". While investigating the male and female human being, Stein puts herself in different points of view: psychological, sociological, historical, philosophical and even teological, which allows her to comprehensively analyse a complex phenomenon envolving human customs, which has been happening for millenia in mankind history: the submission of the feminine. She proposes, on the contrary, equality between both, even in difference. Researching the origins of this phenomenon from the theological point of view, Edith Stein refers to the Scriptures of the Judeo-Christian tradition and revisits the contribution of Genesis and the action of the Redemption of Jesus Christ. It is a proposal that should be considered truly constructive and, in our view, one of the most important in the field of gender studies; in it one can find a convincing critique of inconsistent theories from contemporary culture.
\end{abstract}

Keywords: dual anthropology; feminism; phenomenology; Edith Stein

Si legge nel Vangelo di Giovanni che Gesù:

Lasciò la Giudea e si diresse verso la Galilea. Doveva perciò attraversare la Samaria. Giunse pertanto ad una città chiamata Sicàr, vicino al terreno che Giacobbe aveva dato a Giuseppe suo figlio: qui c'era il pozzo di Giacobbe. Gesù, dunque, stanco del viaggio, sedeva presso il pozzo. Era verso mezzogiorno. Arrivò intanto una donna di Samaria ad attingere acqua. Le disse Gesù: "Dammi da bere". I suoi discepoli intanto erano andati in città a fare provvista di cibi. Ma la Samaritana gli disse: "Come mai tu, che sei Giudeo, chiedi da bere a me che sono una donna samaritana?". I Giudei infatti non mantengono buoni rapporti con i Samaritani. Gesù le rispose: "Se tu conoscessi il dono di Dio e chi è colui che ti dice: Dammi da bere!, tu stessa glielo avresti chiesto e ti avrebbe dato acqua viva" (Gv. 4:3-10).

Si può già iniziare a commentare questo testo e ad osservare che in esso sono descritti i costumi del tempo, secondo i quali gli uomini non potevano parlare in pubblico con una donna estranea. Ci si può chiedere, in primo luogo, perché Gesù non rispetti quest'usanza, anzi addirittura parli con una donna che è sola e che, per di più, appartiene ad un popolo, quello samaritano, che era in contrasto con i Giudei. Con una sola azione Gesù infrange due divieti; ma forse ciò che stupisce maggiormente è che la donna, la quale conosce tali divieti, risponda e si mostri disponibile al dialogo: è curiosità femminile? Anche se fosse, ella si mostra aperta alla novità e il colloquio si avvia sul filo delle domande della donna, che mostra uno straordinario acume, richiamando Gesù alla coerenza con i costumi che avrebbero impedito quel colloquio, chiedendogli di darle l'acqua viva che avrebbe posto fine 
al suo duro lavoro quotidiano di andare al pozzo, e domandandogli, inoltre, come potrebbe procurarsi egli tale acqua dal momento che non possedeva un secchio.

Fin qui l'intelligenza pratica, si potrebbe dire, della donna, la quale, per il momento, sta conducendo il dialogo. Ella rimane sconcertata quando Gesù la chiede di andare a chiamare suo marito, ma non pensa di fingere, al contrario, inizia una sorta di "confessione" da parte di questa donna che dice la verità riguardo alla sua condizione matrimoniale:

"Non ho marito" [e quando Gesù mostra di conoscere la sua vita privata:]
"Hai detto bene "Non ho marito"; infatti, hai avuto cinque mariti e quello
che hai ora non è tuo marito" [anzi la elogia:] "In questo hai detto il vero"
[ella perde la sicurezza mostrata fino ad allora e replica mostrando di aver
identificato la personalità di Gesù:] "Signore, vedo che tu sei un profeta" [e
proprio per questo le sembra opportuno approfittare della situazione
ponendo un quesito di alto valore teologico, così potrebbe essere definito, e
andando alla radice del dissenso fra Samaritani e Giudei:] "I nostri padri
hanno adorato Dio su questo monte e voi dite che è Gerusalemme il luogo in
cui bisogna adorare" (Gv. $4: 17-20)$.

La risposta di Gesù è che con la sua incarnazione il problema del "luogo" in cui si deve adorare Dio viene meno: Dio si adora in spirito e verità, perché Dio è in ogni luogo, anche se la salvezza viene dai Giudei, perché egli è figlio dei Giudei in quanto uomo. Egli rivela alla donna: "Dio è spirito e quelli che lo adorano devono adorarlo in spirito e verità" (Gv. 4: 23); si tratta di una rivelazione "privata" come quella fatta a Pietro e che diventa nota, perché la donna associa le parole di Gesù alla figura del Messia e ottiene piena conferma che Gesù è il Messia. Ella va a raccontarlo ai suoi concittadini, i quali credettero alle sue parole, non rispettando anche loro l'usanza, secondo la quale la testimonianza di una donna era priva di valore. Cade un altro motivo di discriminazione sociale: l'inferiorità giuridica della donna.

Il Vangelo scritto da Giovanni è stato letto, per lo meno dai presbiteri, dagli albori della Chiesa cristiana fino a Giovanni Paolo II, ma egli è il primo che lega espressamente e ufficialmente il racconto al riconoscimento della dignità della donna e della sua parità in quanto essere umano. Certamente egli è il primo che scrive una Lettera Apostolica dall'incipit decisivo Mulieris dignitatem (Giovanni Paolo II, 1988) per porre fine alla discriminazione e rivalutare il ruolo della donna nella società e nella Chiesa, equiparando l'essere umano maschile e quello femminile e andando al di là non solo dei costumi, ma anche di una lunga tradizione presente anche nella Chiesa Cattolica che li confermava, per lo meno dal punto di vista della prassi.

In realtà, probabilmente non era la prima volta che il Vangelo era letto in questa chiave e non solo l'episodio della Samaritana, ma altri luoghi in cui si descrive l'atteggiamento di Gesù nei confronti delle donne. Se si ripercorre a ritroso la letteratura delle donne che hanno riletto i Vangeli per esaminare l'atteggiamento di Gesù, si nota che il Novecento è il secolo più prolifico, ma si è costretti a retrocedere e a risalire alla diffusione della Riforma Protestante, in particolare quella calvinista. Frutti di questa "rivoluzione" si 
trovano nei nascenti Stati Uniti d'America e in Inghilterra nelle comunità puritane. Le donne, alcune delle quali ormai sono in grado di leggere direttamente i testi sacri, senza mediazione dei presbiteri, si rifugiano più che mai nella dimensione religiosa, ma per affermare i loro diritti civili secondo lo spirito della Riforma; in particolare, nella Rivoluzione inglese le comunità riformate davano alle donne un ideale etico della perfezione personale che: "rappresentava l'unica prestazione non legata al sesso, nella quale esse erano in grado di uguagliare, persino di superare gli uomini" (Schulte von Kessel, 1991, p. 172).

Tutto ciò non rimaneva rinchiuso nella pratica religiosa, ma dava la possibilità di trovare la forza per un'azione sociale e politica non solo alle donne appartenenti alle classi sociali elevate, ma anche a quelle, cosa inaudita, appartenenti alle classi inferiori. In Inghilterra esse si espressero attraverso petizioni, ispirate alla teoria dei "livellatori" sostenuta da John Lilburne il quale trasportò nella sfera politica le idee democratiche delle sette religiose; siamo nel 1642. La rivendicazione del diritto di voto da parte degli uomini levellers fu la spinta per una simile richiesta delle donne. La stessa cosa accadde nelle sette puritane dell'East Cost dell'America del Nord; si legge, infatti, nella Storia delle Donne che Abigail Adams chiede a suo marito, delegato del Congresso degli Stati americani, di non dimenticare le rivendicazioni delle donne nel nuovo codice delle leggi se non vuole affrontare una ribellione femminile (Godineau, 1991). Analoghe petizioni sono presentate in Francia da 300 donne parigine che vogliono costituirsi in guardia nazionale nel marzo del 1972.

La risposta degli uomini è sempre la stessa: le richieste sono velleitarie, perché le donne per loro natura "non sono capaci" ad impegnarsi nel sociale. Le donne, però non si scoraggiano, ma il movimento che, soprattutto nella sua genesi era nato su un terreno religioso, si laicizza e si allontana da tutte le chiese. La sordità degli uomini determinerà in gran parte la sorte del femminismo, che diverrà un movimento laico.

Tuttavia, si formano anche alcune associazioni protestanti e cattoliche in Germania, come ci ricorda Edith Stein citando il "Libero Protestantesimo" e il "Movimento Cattolico Tedesco" e 1"“Unione Femminista Cattolica"1 inoltre, singole donne nel mondo cattolico, come Elena Guerra, anche nell'Europa del Sud, cominciano a rivendicare il valore del femminile, ma ciò accadrà più tardi nel XIX secolo.

Tali rivendicazioni ruotano essenzialmente intorno alla questione del riconoscimento dei diritti delle donne sul piano giuridico. Ottima cosa, perché ciò è stato ottenuto nel corso del XX secolo nei paesi occidentali, per lo meno sul piano teorico, altra questione è quella concernente l'effettiva parità per quanto riguarda la prassi e i costumi.

Poiché la diffidenza degli uomini riguardo ad un inserimento effettivo delle donne nella società e la politica era stato sempre legato all'incapacità della "natura femminile" e poiché nel corso dell'Ottocento, ma soprattutto del Novecento avviene anche un mutamento

${ }^{1}$ Si veda una breve storia del movimento femminista rispetto alla Chiese cristiane tracciato dalla Stein (2010) nel testo Problemi della formazione delle ragazze oggi in La donna: questioni e riflessioni. 
sociale della cultura che si diffonde attraverso le scuole e le università in particolare fra la borghesia coinvolgendo anche le donne, alcune di esse manifestano il loro interesse per filosofia e soprattutto per i temi antropologici e cominciano a riflettere sul senso del femminile. Fra queste troviamo, appunto, nel contesto filosofico tedesco Edith Stein.

$\mathrm{E}^{\prime}$ sul contributo teorico che proviene dalle opere di questa pensatrice che intendo soffermarmi, perché è molto approfondito e convincente. La novità della sua impostazione consiste non tanto e non solo nell'aver messo in luce le caratteristiche del "femminile", quanto nell'aver affermato che quest'ultimo non è comprensibile pienamente se non si esaminano le caratteristiche del maschile, perciò ella elabora quella che definisco un'"antropologia duale". Tale elaborazione avviene attraverso il metodo fenomenologico che ella eredita da Edmund Husserl; si tratta di un atteggiamento che tende ad analizzare l'essere umano, come fenomeno che ci viene incontro in noi e negli altri.

E'chiaro che, assumendo un atteggiamento filosofico, qual è quello fenomenologico, ciò che, in primo luogo, si mette in evidenza è la struttura universale dell'umano, analizzato in particolare sulla base della propria esperienza personale, convalidata, però, dal ritrovamento delle stesse strutture nell'alterità. L'universalità della struttura si articola, tuttavia, nella singolarità di ciascuno, pertanto, è opportuno esaminare tale singolarità, nella quale quella è presente, ma declinata in modo assolutamente personale.

Questo è l'obiettivo di Edith Stein. Ella sulle orme del suo maestro Husserl, del quale ha trascritto i manoscritti riguardanti l'analisi dell'essere umano, confluiti nel secondo volume delle Idee per una fenomenologia pura e una filosofia fenomenologica, conduce una capillare descrizione dell'umano.

\section{Dall'universale al duale}

Le analisi, che sono state condotte nella filosofia occidentale, hanno individuato prevalentemente una struttura comune a tutti gli esseri umani, struttura che prescinde dalle situazioni storiche, sociali, culturali, nelle quali il singolo è inserito ed anche dalle connotazioni di genere. $\mathrm{E}^{\prime}$ stato merito del pensiero femminista scendere dal piano dell'universalità a quello, per lo meno intermedio, della differenza di genere, e procedere ad un'ulteriore analisi (Ales Bello, 2017)2.

Se si vuole, tuttavia, valorizzare il piano esperienziale, che è quello su cui quotidianamente viviamo, è necessario osservare che le donne che incontriamo - ed anche gli uomini - si presentano nella loro singolarità; infatti, non incontriamo mai la donna o l'uomo nella sua astrattezza, ma sempre una persona con sue caratteristiche peculiari: questo è un dato incontrovertibile della nostra esperienza. Tuttavia, quando si riflette sulla differenza di genere e, in particolare, sul femminile, anche il linguaggio ci spinge a

\footnotetext{
2 Si veda in particolare la Prima Parte, La nascita del femminismo e la questione del gender
} 
generalizzare e, quindi, a parlare della "donna", ricercando proprio gli elementi caratteristici presenti in ogni donna.

Quest'atteggiamento è privilegiato e considerato fondamentale nell'ambito della ricerca che chiamiamo filosofica, la quale tende verso la messa in evidenza di strutture universali, quelle che, d'altra parte, riconosciamo presenti quando, incontrando una donna o più donne, diciamo che sono donne. Ma che cosa in verità 'riconosciamo'? Esse ci vengono incontro con una struttura fisica determinata, che già porta con sé e ci propone un paradosso: ognuna di loro è unica e irripetibile, eppure sono tutte donne.

Si deve costatare, in verità, che possediamo la capacità di andare dalla singolarità all'universalità e viceversa. Questa possibilità, che c'è data, presenta grandi vantaggi e altrettanti rischi; infatti, se ci si ferma all'evidenziazione dell'universalità ci sfugge la singolarità e il piano esperienziale, che ci dà il contatto diretto con l'oggetto - che è trascurato se non occultato -, se, però, ci fermiamo alla singolarità, perdiamo di vista i tratti comuni, il cui rilevamento può essere valido per un migliore orientamento teorico. Nella capacità di tener presenti i due momenti nella loro complementarità e ineliminabilità risiede l'equilibrio, che può dare risultati fecondi nel "pensare l'esperienza".

La singolarità si sente, si afferra attraverso la consapevolezza di sé e l'entropatia nei confronti dell'altro. Uso il termine entropatia per render in italiano il sostantivo tedesco Einfühlung, perché il vocabolo empatia, che serve anche a tradurlo, ha assunto comunemente una connotazione non fedele con l'indicazione dei fenomenologi, in particolare di Edmund Husserl ed Edith Stein, i quali lo hanno usato per primi in senso filosofico per indicare il riconoscimento intuitivo dell'alterità. Certamente tale riconoscimento può esser la via per una comprensione più profonda dell'altro e uno stare vicino all'altro, tuttavia, quest'ultimo atteggiamento manifesta altre modalità del vivere che coinvolgono la simpatia, l'attenzione, la benevolenza, l'amore: tutti momenti che possono essere vissuti in concomitanza, ma richiedono, in primo luogo, l'originario riconoscimento dell'alterità e la nostra capacità di avere esperienze vissute. Si tratta degli Erlebnisse, dei quali per primo parla Husserl indicandoli come gli strumenti da noi posseduti potenzialmente che ci mettono in contatto con noi stessi e con le cose del mondo esterno. Ed è proprio l'entropatia - intesa com'è stata proposta dalla scuola fenomenologica, consistente nel vissuto attraverso il quale riconosciamo l'alter ego, simile a me nelle strutture essenziali, ma unico nelle sue specificazioni personali - che ci consente di fare il salto verso l'universalità, in quanto si tratta dell'intuitiva e immediata messa in evidenza di ciò che è comune, pur nella particolarità dell'esperienza vissuta all'interno della propria irripetibile singolarità.

L'impatto esperienziale con me stesso e con l'altro passa, in primo luogo, attraverso la corporeità.

Questo fatto ci spinge ad analizzare la corporeità, della quale pertanto non si può fare a meno, e sappiamo che la cultura occidentale, attraverso l'approccio scientifico, ha cercato di penetrare all'interno della corporeità stessa esaminandola in molteplici prospettive sotto il 
profilo della fisiologia, dell'anatomia e della genetica e mostrando, in tal modo, le caratteristiche del corpo femminile e maschile, che si articolano, poi, in modo particolare in ogni donna e ogni uomo. Su tale base, come sappiamo, opera la medicina, cogliendo le alterazioni e procedendo alla terapia ed è proprio quest'ultima che si trova a fare i conti, in modo qualche volta difficile, se non drammatico, con la singolarità.

Certamente è stato merito del femminismo l'aver messo in evidenza la necessità di distinguere la donna dall'uomo e di non fermarsi al neutro. A questo proposito si pongono due questioni, la prima riguarda il fatto che la donna ha sì una particolare configurazione anatomica, ma che anche molte sue caratteristiche sono in comune con l'altro essere umano che è l'uomo; infatti, si parla in generale di corpo umano che va aldilà delle specificazioni del femminile e del maschile. Proprio questa constatazione ci conduce alla seconda questione: se non si può analizzare la donna senza tener conto che è un essere umano, bisogna correlativamente esaminare anche l'uomo.

Già in questo primo stadio della ricerca si delinea una gerarchia che può essere percorsa dal basso o dall'alto, ma in ogni caso indica la correlazione dei due momenti; infatti, l'essere umano è articolato nel maschile e nel femminile ed un'analisi corretta ci costringe a tener presenti entrambi gli aspetti.

Come teorizzare tutto questo da un punto di vista più ampio che possiamo definire filosofico? Proprio l'esame del singolo ci rimanda all'universalità dell'essere umano, ma di nuovo siamo costretti a scendere verso la partizione quella del maschile e del femminile, prima di giungere alla singolarità. In realtà, come si è sopra accennato, è possibile percorrere anche il cammino inverso, e, quindi, i due cammini sono correlativi e circolari.

Tutto ciò, prima di proseguire l'analisi dell'essere umano nelle sue articolazioni femminile e maschile, fa riflettere sul fatto che, se si vuole procedere ad un'analisi dell'essere umano, quindi, ad un'antropologia, quest'ultima deve essere anche un'antropologia duale. Infatti, se è vero che si possono indicare alcuni elementi universali che distinguono, ad esempio, l'essere umano da quello animale o vegetale, un necessario approfondimento della struttura umana ci conduce a cogliere la dualità come elemento importante e imprescindibile dell'analisi.

Questo tema è stato affrontato con acutezza teoretica da Edith Stein, la quale, venuta a conoscenza del movimento femminista durante la frequenza dell'Università, riflette sulle basi filosofiche necessarie per una seria impostazione della questione.

\section{Dualità e sessualità}

La differenza fra uomo e donna passa in primo luogo attraverso la corporeità sessuata. Allora è opportuno chiedersi che cosa sia la sessualità. Il corpo vivente è definito come un corpo psico-fisico e l'essere umano come un'entità complessa, in cui è presente anche la dimensione dello spirito. Se si affronta la questione della sessualità umana non ci si può 
limitare alla trattazione del corpo dal punto di vista anatomico, ma bisogna prendere in considerazione l'essere umano nella sua totalità.

La Stein (2010) nelle sue conferenze dedicate alla donna e al suo rapporto con l'uomo ora raccolte nel libro La donna: questioni e riflessioni ha parlato di una doppia specie che si articola nel femminile e nel maschile includendo tutte le componenti dell'umano. E ciò è espresso ai nostri giorni con il termine preso dalla lingua anglosassone, "genere". La Stein, seguendo l'indicazione aristotelica, ha distinto genere umano e specie maschile e femminile, ma queste distinzioni sono ormai lontane dalla mentalità prevalente nella cultura occidentale contemporaneo. Il termine genere sostituisce quello di che la Stein chiamava specie, che per lei era connessa anche al sesso. Ella francamente si riferisce al sesso maschile e al sesso femminile, senza alcuna intenzione riduttiva, ma intendendolo in modo ampio come comprendente tutto l'umano nelle sue differenze. E' chiaro, allora, che la sessualità non riguarda solo l'aspetto fisiologico ma coinvolge anche la dimensione psichica e quella spirituale.

Non separando alcuna delle componenti dell'umano si può sostenere che l'educazione e la formazione ${ }^{3}$, che sono indispensabili per la vita umana, contengono quella che è definita "educazione sessuale". Essa consiste nella ricerca dell'equilibrio fra i diversi aspetti costitutivi dell'uomo e della donna, dando certamente uno spazio notevole alla capacità umana di controllo libero e motivato che si genere nello spirito, ma senza sottovalutare la dimensione psichica, quella caratterizzata dalle tendenze, dagli impulsi, dagli istinti, che - ella sostiene - ci "capitano", cioè non sono da noi provocati, ma sono da noi scoperti presenti in noi stessi.

Pur non potendo prescindere dalla complessità dell'umano, è possibile indagare analiticamente le sue varie componenti ed è anche possibile elaborare una disciplina come la psicologia, la quale non può prescindere da una visione globale dell'umano. Questo messaggio si trova nelle opere husserliane ed anche nelle indagini steiniane (Ales Bello, 2016).

\section{Aspetti corporei e psichici della sessualità}

Il merito della Stein, come si è già detto, sta nell'aver non solo considerato l'essere umano nella sua universalità - cosa sempre legittima, ma di averlo studiato nella sua articolazione maschile e femminile. Si tratta, in fondo, sempre di un' universalizzazione che, però, non parte dal "neutro", ma dal "duale". Ella indica, in primo luogo, le differenze anatomiche presenti nell'identificazione dei sessi, anche se su di esse non si sofferma in

\footnotetext{
3 Educazione e formazione sono distinte molto acutamente da Edith Stein (2017) nei suoi scritti dedicati alle questioni pedagogiche ora raccolti in Formazione e sviluppo dell'individualità. La distinzione consiste nella funzione prevalentemente "informativa" dell'educazione che deve essere completata da un progetto esistenziale che solo la formazione può aiutare ad elaborare.
} 
modo specifico poiché non è questo il suo obiettivo e lascia il campo all'anatomia. Maggiormente analizza gli aspetti psichici, le tendenze che distinguono i sessi: unidirezionalità del maschile e completezza del femminile rappresentano le due caratteristiche fondamentalmente riscontrabili a livello psichico, ma presenti anche a livello anatomico e spirituale.

L'uni direzionalità maschile, che è facilmente individuabile dal punto di vista anatomico, è riscontrabile anche dal punto di vista psichico perché riguarda una tendenza a concentrarsi su un elemento specifico, ad agire prevalentemente avendo di mira una meta, escludendo tutto il resto. Rispetto all'apertura verso altre direzioni, in realtà siamo di fronte ad una "chiusura". La Stein attribuisce al femminile la "completezza". Questo termine deve essere bene inteso: si tratta di una situazione che si realizza alla fine di un movimento che è quello dell'apertura per l'accoglienza e di chiusura in una sorta di abbraccio. Quindi di un movimento avvolgente di protezione, un movimento essenzialmente "materno".

Il maschile fissa l'attenzione su uno o alcuni aspetti escludendo gli altri, il femminile si allarga a comprenderli tutti. Il femminile mostra, perciò, di voler favorire ciò che é l'umano nella sua interezza, il maschile di portare l'umano alle sue massime prestazioni al prezzo di esclusioni.

\section{La dualità dal punto di vista teologico}

Finora si è parlato della differenza fra uomo e donna dal punto di vista filosofico e psicologico, punti di vista fondamentali per la Stein, ma quando si affronta la questione da un altro punto di vista, quello che ha determinato la nascita stessa del femminismo, cioè la valutazione del maschile e del femminile, connessa alla lunga storia della discriminazione delle donne considerate inferiori, ella sostiene che la questione è da porsi sul piano squisitamente morale e per comprenderla è necessario risalire al peccato originale. Ella teorizza, pertanto, un approccio metodico, che definisce teologico, affrontando l'origine del disordine morale.

Si tratta di esaminare, in primo luogo, gli scritti dei Dottori e dei Padri della Chiesa, se si vuole rimanere nell'ambito strettamente teologico; ella afferma che il materiale è abbondante, tuttavia è "un materiale rispetto al quale esiste la possibilità della critica" (Stein, 2010, p. 217). La Stein non si sofferma, come fa, ad esempio, K. E. Borresen ad esaminare tutti i testi di Agostino e Tommaso in cui si parla della "natura" della donna, in particolare sull'interpretazione della donna proposta da quest'ultimo come mas occasionatus (Borresen, 1979), uomo mancato, le basta citare una frase meno aggressiva, ma a suo avviso, altrettanto negativa: "Vir est principium mulieris et finis" per chiedersi "che senso abbia quest'affermazione e da quale fonte derivi" (Stein, 2010, p. 217) e ancora: 
Si dovrebbero inoltre esaminare tutti i passi scritturistici da cui è possibile ricavare una definizione della finalità della donna (e, in modo analogo, qualcosa della sua posizione "secondaria" nei confronti dell'uomo); e confrontare ciò che se ne ricava quanto a principio e fine con quell'affermazione di Tommaso (idem).

I testi citati sono tratti dal saggio Problemi della formazione delle ragazze oggi, manoscritto del corso che la Stein tenne nel semestre estivo 1932 presso l'Istituto tedesco di Pedagogia Scientifica, un' istituzione cattolica, di Münster. Mi sembra che la risposta alle domande sopra poste si trovi in una conferenza tenuta nell'ottobre del 1931 presso le Orsoline di Aquisgrana dal titolo Vocazione dell'uomo e della donna secondo l'ordine della natura e della grazia, in cui commenta l'origine dell'uomo e della donna esaminando i passi del Genesi.

Ella è fra le prime studiose a mettere in correlazione i due passi 1,26-29 e 2,27-25, cosa che farà Giovanni Paolo II nella Lettera Apostolica Mulieris dignitatem. La Stein (2010) nota una sostanziale concordanza fra i due testi, nel senso che nel primo si parla di una nascita contemporanea dei due esseri umani, ai quali Dio affida un triplice compito: essere immagine di Dio, generare una discendenza e dominare la terra e questo compito è affidato ad entrambi, posti su un piano di parità assoluta. Il secondo racconto che all'inizio sembra dare maggiore importanza all'uomo, giacché creato per primo, in realtà, nel prosieguo non sottolinea una disparità, al contrario, traendo dall'uomo la donna gli diede un "aiuto a lui dirimpetto" cos' ella traduce l'espressione ebraica eser kenegdo, intendendo: "un completamento, un pendant, così che entrambi si assomigliano, per quanto non in tutto, ma in modo da completarsi a vicenda come una mano rispetto all'altra" (p. 82). Il commento finale mi sembra particolarmente significativo: anche in questo caso si trova un'immagine di Dio, infatti,

Dio è uno e trino: come il Figlio procede dal Padre e dal Padre e dal Figlio lo Spirito, così la donna è uscita dall'uomo e da ambedue discendono i posteri. E ancora: Dio è amore. Ma tra meno di due non vi può essere amore (come dice san Gregorio nell'omelia sulla missione dei discepoli, mandati a due a due) (Stein, 2010, p. 83).

Ella accetta che Eva nasca dalla costola, ma se quel termine significasse, come sembra che significhi nella lingua ebraica, solo il fianco e il lato, si tratterebbe di una sorta di divisione di Adam in Adamo ed Eva.

Tuttavia, anche in questo caso non si può parlare, secondo la Stein, di dominio dell'uomo sulla donna perché ella è compagna e aiuto. Quando si è generato lo squilibrio fra i due? Certamente in occasione del peccato; infatti, in occasione del mutato rapporto con Dio, si attua un mutato rapporto fra i due esseri umani. La Stein ritiene che il peccato riguardi la sfera della procreazione e dice che, anche se non conosciamo la modalità della procreazione prima del peccato, la conoscenza del bene e del male, promessa dal serpente, condusse i due esseri umani a guardarsi con occhi diversi e persero l'innocenza nei loro reciproci rapporti. 
Perciò il peccato non è solo una generica disobbedienza, ma "un tipo di unione reciproca che contraddiceva l'ordine originario" (Stein, 2010, p. 85). Conoscere il male significava in questo caso fare il male, perché anche prima del peccato, a suo avviso, gli esseri umani sapevano la differenza fra bene e male, ma non conoscevano il male perché non lo avevano fatto: avrebbero dovuto "guardarsi da quella conoscenza del male che si acquisisce commettendolo" (idem).

Si potrebbe aggiungere al commento della Stein che il grande inganno del demonio è stato quello di assicurare che se avessero conosciuto il bene e il male in senso pieno, sarebbero diventati come Dio; infatti, a che cosa aspira l'essere umano? A conoscere tutto e perché ciò accade? Perché ha in sé una traccia dell'Assoluto e, quindi, ne ha una conoscenza, benché vaga, ma nello stesso tempo si rende conto di essere finito. Se non accetta con umiltà la sua condizione di finitezza, nasce in lui e sembra che per la prima volta sia nato in lei, in Eva, il desiderio smodato di essere come Dio: si tratta del peccato di superbia, peccato certamente condiviso da Adamo.

Questa è l'origine della condanna di Dio e del conflitto fra i due esseri umani, tuttavia, contemporaneamente Egli apre la porta della speranza dicendo: “Io porrò inimicizia tra te e la donna, tra la tua stirpe e la sua stirpe: questa ti schiaccerà la testa e tu le insidierai il calcagno" (Gen 3,15). L'osservazione della Stein a tale proposito è molto interessante, perché ella certamente accetta che Dio qui ci si riferisca a Maria, Madre di Dio, ma

Questo non esclude però l'altro senso: che già alla prima donna, cui Adamo diede il nome di "madre di tutti i viventi" e a tutte quelle che l'avrebbero seguita venisse affidato come compito particolare la lotta contro il male e con ciò, la preparazione del recupero della vita (Stein, 2010, p.86).

La prova dell'affidamento di questo compito si trova, secondo la nostra pensatrice, nelle parole che Eva pronunciò quando nacque il suo primo figlio: "Ho acquistato un uomo dal Signore", con le quali riconosce una benedizione del Signore ed è per questo che le donne d'Israele hanno sempre voluto procreare per preparare la generazione che avrebbe visto la salvezza, la Redenzione di Gesù, figlio di Maria.

La Redenzione ha introdotto nella storia la possibilità del superamento del contrasto fra uomo e donna, se ci si lascia guidare dalla grazia di Dio: questo è il messaggio di speranza che la Stein coltiva e che propone di divulgare attraverso un'efficace opera educativa. A tale opera la Stein dedica le sue conferenze sulla donna e scritti di carattere pedagogico, ancora oggi di grande attualità (Stein, 2017).

La trattazione del metodo teologico contiene un'altra preziosa indicazione utile per la comprensione di ciò che accade nella cultura occidentale contemporanea. L'argomentazione è molto interessante, perché verte sul rapporto fra "natura" ed "essenza" riguardo all'uso dei due termini che si trovano negli scritti di Tommaso d'Aquino. Per comprenderla è opportuno ricordare la formazione filosofica di Edith Stein. La fenomenologia di Husserl, 
alla quale ella aveva aderito in gioventù, si distingueva dalla corrente di pensiero dominante in Europa tra la fine dell'Ottocento e i primi decenni del Novecento: il Positivismo. Questa corrente fissava la sua attenzione sulla "natura" letta in termini fisico-matematici e tendeva ad assolutizzarla identificandola con tutta la realtà. Si proponeva, pertanto, un'interpretazione naturalistica, contro la quale alcuni filosofi presero posizione e fra questi, appunto, Husserl.

Per tale ragione, il termine "natura" delle cose, usato ad esempio da Tommaso d'Aquino, non si trova nei testi di Husserl, che parla invece dell'“essenza" delle cose. Con il termine "natura" Husserl intendeva soltanto la natura nel senso fisico. La Stein, nella fase strettamente fenomenologica, seguendo Husserl, utilizza il termine essenza, per comprendere il "senso" dei fenomeni che sono sottoposti a indagine. Quando ella, però, si avvicina al pensiero medievale e, in particolare, a quello di Tommaso d'Aquino, si rende conto che per lui l'essenza delle cose è definita anche "natura" delle cose, senza alcun riferimento alla natura fisica, indicandone, appunto, l'intima struttura.

Sulla scia di tale constatazione scrive: “In Tommaso 'natura' ed 'essenza' vengono spesso utilizzati come sinonimi. Entrambe indicano ciò che una cosa è in se stessa" (Stein, 2010, p. 218). Tuttavia, osserva:

Questo "che cosa", però, può essere colto da diversi punti di vista: in un primo senso come ciò che viene assegnato alla cosa all'atto della creazione, come ciò che lo qualifica nel suo esser posto in essere e che ne determina l'agire. E a ciò si addice il termine natura. Poi, come ciò che troviamo nella cosa come quel che le è proprio e necessario, rispetto a tutte le qualità esterne e ai modi di darsi e di apparire che dipendono dai condizionamenti accidentali ed esterni della sua esistenza. E a ciò si addice il termine essenza (idem).

Si nota che la Stein sembra qui non accettare fino in fondo l'equivalenza proposta dai pensatori medievali e distingue la struttura essenziale dalla realizzazione empirica delle realtà esistenti, sostenendo che, se si vuole cogliere la struttura di una cosa dal punto di vista conoscitivo, dobbiamo individuarne l'essenza, cioè il suo senso. La realtà esistente è una realizzazione dell'essenza che può contenere in sé variazioni particolari. A questo proposito, la Stein sta riproponendo il tema husserliano della variazione d'essenza, cioè il fatto che in ogni realtà empirica si trova un momento essenziale, che può essere colto al di là delle modalità concrete in cui si presenta; si tratta dell'invariante rispetto a tutte le variazioni, che sono tali proprio perché è presente l'invariante stessa. La conoscenza umana coglie anche le variazioni, ma se si deve dire che cosa è una cosa, è nelle possibilità umane afferrarne il senso.

Applicando tutto ciò all'indagine sulla donna, la sua natura, così com'è legata alla sua vocazione, cioè al compito particolare che le compete, può presentarsi come una variazione senza che l'essenza ne sia toccata. Dal punto di vista teologico, ciò che interessa è il fine della donna, appunto la sua vocazione, quindi la sua natura. 
La Stein riconosce che la questione dell'essenza è puramente filosofica e non teologica; infatti, riguarda la struttura del femminile in quanto tale. La differenza fra i due punti di vista, teologico e filosofico, a suo avviso non impedisce che possano essere considerati entrambi validi.

Di là dall'impostazione filosofica o teologica, la distinzione proposta dalla Stein fra natura ed essenza può essere una chiave interpretativa importante riguardo alle questioni sollevate nella cultura occidentale contemporanea sul tema del "genere" e del "gender".

La non identificazione tra natura ed essenza mi sembra aggiungere un tassello ulteriore all'indagine; infatti, poiché non si possono disconoscere i casi di omosessualità, trans-sessualità, intersessualità, come giustificarli rispetto al binarismo maschile/femminile, se non si procede alla distinzione fra essenza e natura?

In primo luogo, direi che fondamentale è l'interpretazione fenomenologica della tripartizione dell'essere umano in corpo, psiche e spirito, che bisogna tener presente quando si affrontano tali argomenti. E anche l'unità psicofisica del corpo vivente (Leib), orientato dallo spirito. Come si lega tutto ciò al rapporto fra natura ed essenza? Propongo di leggere i fenomeni sopra descritti nel modo seguente: femminile e maschile costituiscono due poli ideali che possono essere più o meno perfettamente realizzati nella natura. La Stein, sostenendo ciò, si riferiva all'essenza del femminile e alla vocazione di ogni singola donna, nonché alla sua vocazione personale.

Si può applicare tutto ciò alle situazioni individuali sotto il profilo della sessualità. La "natura", nel senso proposto in questo caso dalla Stein, è la realizzazione del singolo: è possibile che non ci sia armonia fra le parti che lo compongono, ad esempio, fra corpo e psiche? La "natura", così intesa, può non attuare l'essenza; ciò implica che la disarmonia fra le parti non rispetta l'ideale. Potrebbero manifestarsi particolari dosaggi ormonali, disturbi della differenziazione gonadica (ovaia e testicolo) e anche in quella fenotipica (organi della riproduzione), ad esempio. Ma, allora, l'essenza viene meno? No, perché il bipolarismo è indicato come punto di riferimento in biologia, in medicina e, conseguentemente, nel diritto. Pur riconoscendo in alcuni soggetti ambiguità nella determinazione sessuale dal punto di vista genetico ed endocrinologico, si procede ora con cautela negli interventi, che, apparentemente, potrebbero risolvere tale ambiguità, perché l'orientamento sessuale riguarda tutta la complessa struttura umana, direbbe la Stein, e potrebbero risvegliarsi in una direzione diversa da quella scelta ${ }^{4}$.

\footnotetext{
${ }^{4}$ Ho sviluppato questi argomenti nel sopra citato Tutta colpa di Eva: antropologia e religione dal femminismo alla gender theory (Ales Bello, 2017). Si leggano, inoltre, i contributi di Carlo Cirotto (2015), Identità di genere: considerazioni di un biologo; quello di Antonio Balsamo (2015), Genetica e sviluppo della sessualità, e la riflessione di Paolo Cavana (2015), Questione del "genere", diritti del minore e procreativa in Natura e cultura nella questione del genere (Facchini, 2015). Questo argomento è anche trattato da Laura Palazzani (2016) nel suo saggio I disturbi della differenza sessuale e l'intersessualità: una questione gender tra teoria e prassi, in Desideri, figli, gender, a cura di Borghesi, Palazzani e Pessina (2016).
} 
Diverso è il caso di quell'aspetto della teoria queer che, in modo provocatorio nei confronti di cosiddetti conformismi sociali e in mancanza di ambiguità palesi, attribuisce alla volontà umana la capacità di scegliere in modo arbitrario; paradossalmente questa è una conferma del valore della libertà umana, da attribuirsi all'attività dello spirito, pur essendo i motivi che spingono a tale condotta legati a reazioni psichiche, in opposizione a tradizioni e costumi considerati opprimenti. È opportuno osservare che $\mathrm{i}$ costumi non sempre costituiscono una regola da seguire e devono essere anch'essi vagliati alla luce di criteri non dettati da reazioni e risentimenti, piuttosto da una riflessione consapevole che segua criteri universali.

Il fatto che maschile e femminile rappresentino i due poli ideali rispetto all'essenza dell' essere umano e non - come sostiene anche Judith Butler (2014) - costruzioni puramente sociali, non conduce all'esistenza di un terzo genere neutro, ma alla possibilità di diverse realizzazioni del bipolarismo stesso. Questo mi sembra un punto importante, perché, senza mettere radicalmente in crisi l'antropologa duale, la Stein riconosce che il "dosaggio" fra i due sessi, non essendo mai stabilito in modo definitivo, può subire variazioni nel singolo caso.

Per quanto concerne il tema della singolarità, è interessante notare che essa è particolarmente esaltata dalle prese di posizione contemporanee non solo relative al gender ed è presente anche nelle altre teorie sul genere, ancorate tutte prevalentemente all'arbitrio individuale, piuttosto che all'identità profonda del soggetto.

A questo punto, per scavare ulteriormente su questo tema così com'è proposto da Edith Stein, ci si può chiedere se nel nucleo identitario, che costituisce a suo avviso il perno della persona, sia iscritta l'essenza maschile o femminile e il suo orientamento "naturale".

La Stein non affronta tale argomento; tuttavia, a favore di una risposta positiva al quesito giocherebbe il fatto che il nucleo è il luogo in cui è presente il destino individuale, quindi, anche l'orientamento sessuale del corpo, della psiche e dello spirito, tutto ciò da un punto di vista filosofico, ma anche perché l'essere umano è stato creato a immagine di Dio come uomo e donna, com'è detto nell'Antico Testamento. D'altra parte, pur essendo la sessualità una componente importante dell'essere umano, il nucleo, come anima dell'anima, supera tutto ciò, perché è il momento di eternità presente nell'umano e, quindi, sembra trascendere ogni connotazione naturale, pur orientando la "natura". La questione, pertanto, è difficilmente risolvibile con gli strumenti offerti dall'Autrice, anche se si può propendere per la prima ipotesi.

\section{Una sintesi del percorso}

Si è detto come la Stein s'inserisce nel movimento femministe - sopra descritto nelle sue linee essenziali - con una sua originalità che consiste, in primo luogo, nell'aver posto la questione del femminile in un'ottica filosofica e, conseguentemente, nell'aver indicato la 
necessità della trattazione dell'essenza dell'essere umano sia nei suoi aspetti universali, sia nella dualità del maschile e del femminile; in secondo luogo, di aver fatto riferimento all'opportunità di affrontare la differenza anche da un punto di vista psicologico, e in terzo luogo, di aver considerato fondamentale risalire all'origine del conflitto che si costata fra i due sessi e, quindi, affrontare la loro origine nella prospettiva teologica. Secondo quanto ella ci propone, mi sembra che l'indagine dovrebbe essere rovesciata, perché ciò che giustifica in ultima analisi tutto ciò che concerne l'umano è la sua condizione di creatura e la sua disobbedienza a Dio che introduce il male nel mondo e determina la conflittualità fra gli esseri umani.

La speranza nella Redenzione è certamente la via d'uscita, ma non automatica, essa richiede un impegno attivo da parte degli umani. Ciò è testimoniato dal fatto che senza il riconoscimento del messaggio cristiano e l'adesione concreta a Cristo, gli esseri umani perpetuano forme di aggressività reciproca.

La Stein ci aiuta, inoltre, a comprendere non solo la dualità umana, ma anche le "variazioni" rispetto alla dualità: chissà che cosa avrebbe detto se avesse conosciuto le interpretazioni che si definiscono "teologiche" di coloro che intendono radicalmente mettere in crisi la dualità in nome di una "libertà" senza confini come accade nella teologia queer. Noi possiamo tentare di porci sul solco delle sue proposte per una presa di posizione, equilibrata e costruttiva.

\section{Riferimenti}

Ales Bello, A. (2016). Il senso dell'umano: tra fenomenologia, psicologia e psicopatologia. Roma: Castelvecchi.

Ales Bello, A. (2017). Tutta colpa di Eva: antropologia e religione dal femminismo alla gender theory. Roma: Castelvecchi.

Balsamo, A. (2015). Genetica e sviluppo della sessualità. In F. Facchini (Ed.). Natura e cultura nella questione del genere (pp. 67-83). Bologna, Italia: EDB.

Borresen, K. E. (1979). Natura e ruolo della donnain Agostino e Tommaso. Assisi, Italia: Cittadella.

Butler, J. (2014). Fare e disfare il genere (F. Zappino, Trad.) Milano: Mimesis.

Cavana, P. (2015). Questione del "genere", diritti del minore e procreativa. In F. Facchini (Ed.). Natura e cultura nella questione del genere (pp. 105-125). Bologna, Italia: EDB.

Cirotto, C. (2015). Identità di genere: considerazioni di un biologo. In F. Facchini (Ed.). Natura e cultura nella questione del genere (pp.49-65). Bologna, Italia: EDB.

Giovanni Paolo II (1988). La dignità della donna: lettera apostolica "Mulieris dignitatem". Roma: Elledici. 
Godineau, D. (1991). La frattura politca e il nuovo ordine del discorso - sulle due sponde dell"Atlantico: pratiche rivoluzionarie femminili. In G. Duby \& M. Perrot. (Ed.s). Storia delle donne in occidente, IV: l'Ottocento (pp. 15-33). Roma: Laterza.

Palazzani, L. (2016). I disturbi della differenza sessuale e l'intersessualità: una questione gender tra teoria e prassi. In M. Borghesi. (Ed.) Desideri, figli, gender (pp. 362-381). Roma: Studium.

Schulte von Kessel, E. (1991). Vergini e madri fra cielo e terra: le cristiane nella prima età moderna. In G. Duby \& M. Perrot. (Ed.s). Storia delle donne in occidente, III: dal Riniscimento all'Età Moderna (pp. 156-200). Roma: Laterza.

Stein, E. (2010). La donna: questioni e riflessioni. (O. Nobile, Trad.; A. M. Pezzella \& M. Paolinelli, Rev.; A. Ales Bello \& M. Paolinelli, Ed.s). Roma: Città Nuova.

Stein, E. (2017). Formazione e sviluppo dell'individualità (A. M. Pezzella \& A. Togni, Trad.s; A. Ales Bello, Pref.). Roma: Città Nuova.

\section{Nota sobre a autora}

Angela Ales Bello é Doutora em Filosofia, professora emérita de História da Filosofia na Pontificia Università Lateranense em Roma, Itália. É Presidente do "Centro Italiano di Ricerche Fenomenologiche" - filiado a "The World Phenomenology Institute" (E.U.A.) - e da "Associazione Italiana Edith Stein". Faz parte do comitê de redação de numerosas revistas internacionais, dentre as quais "Phenomenological Inquiry" (E.U.A). Suas publicações se referem à relação entre fenomenologia alemã e outras correntes do pensamento contemporâneo na perspectiva histórica e teorética. É co-editora da tradução italiana da Obras Completas de Edith Stein. Email: alesbello@tiscali.it 